Research Article

\title{
Surface Laser Quenching as an Alternative Method for Conventional Quenching and Tempering Treatment of 1538 MV Steel
}

\author{
R. Carrera-Espinoza $\left(\mathbb{D},{ }^{1,2}\right.$ A. Rojo Valerio, ${ }^{3}$ J. del Prado Villasana, ${ }^{4}$ J. A. Yescas Hernández, ${ }^{2}$ \\ P. Moreno-Garibaldi, ${ }^{2}$ M. A. Cruz-Gómez, ${ }^{5}$ and U. Figueroa López ${ }^{1}{ }^{1}$ \\ ${ }^{1}$ Escuela de Ingeniería y Ciencias, Tecnológico de Monterrey, Carr. Lago de Guadalupe Km. 3.5, Margarita Maza de Juárez, \\ 52926 Atizapán de Zaragoza, Mexico \\ ${ }^{2}$ Universidad de las Américas Puebla, Ex Hacienda Sta. Catarina Mártir S/N, San Andrés Cholula, Puebla C.P. 72810, Mexico \\ ${ }^{3}$ Escuela de Ingeniería y Ciencias, Tecnológico de Monterrey, Eduardo Monroy Cárdenas 2000, San Antonio Buenavista, \\ 50110 Toluca de Lerdo, Mexico \\ ${ }^{4}$ Investigación Desarrollo e Innovación, MACIMEX, Carr. La Marquesa Tenango, 52300 Tenango del Valle, Mexico \\ ${ }^{5}$ Benemérita Universidad Autónoma de Puebla, Facultad de Ingeniería, Tribology and Transportation Group, \\ Edificio de Posgrado, Primer nivel, Cubículo Núm, 16, Blvd. Valsequillo Esq. Av. San Claudio, Ciudad Universitaria, \\ Col. San Manuel, C.P., 72570 Puebla, Mexico
}

Correspondence should be addressed to R. Carrera-Espinoza; rafaelcb05@gmail.com and U. Figueroa López; ufiguero@itesm.mx

Received 26 September 2019; Revised 10 February 2020; Accepted 5 March 2020; Published 1 April 2020

Academic Editor: Stanislaw Dymek

Copyright (C) 2020 R. Carrera-Espinoza et al. This is an open access article distributed under the Creative Commons Attribution License, which permits unrestricted use, distribution, and reproduction in any medium, provided the original work is properly cited.

\begin{abstract}
This paper aims at encouraging the use of laser treatment as an environmentally friendly technique to improve the mechanical properties of metallic materials over conventional quenching and tempering techniques through the study of the tribological behavior of AISI $1538 \mathrm{MV}$ steel subjected to surface laser quenching treatment. Sliding wear tests were carried out by the pin-ondisk method. In order to identify the wear mechanisms, the worn surfaces on the disks were evaluated by scanning electron microscopy and the wear scars on the ball were observed by optical microscopy. Results reveal that laser treatment reduces the average friction coefficient by $25 \%$ and the wear rate by $60 \%$ compared with those achieved by the conventional methods, while the depths of the wear track and hardness of the cross section and surface are maintained.
\end{abstract}

\section{Introduction}

Heat treatment involves a collection of processes that aim to improve the mechanical properties of metallic materials by modifying their initial crystalline structure. One of these processes is conventional quenching and tempering (CQT) treatment, which is used to increase the hardness of steelbased mechanical components that are expected to withstand high surface loading. The basic principle of conventional quenching of ferrous materials is the heating of the working material up to the austenitization temperature followed by abrupt cooling to obtain the martensitic phase, which confers the mechanical components with high hardness and wear resistance. One of the major disadvantages of conventional quenching is that the entire component undergoes phase change, even though only a surfacehardened layer is required at specific locations in some machine components with the main body left intact.

Several surface hardening techniques have been developed and tested on metals commonly used in the automotive industry to manufacture gears, valves, sprockets, cams, gear housings, crankshafts, and cylinder liners among others. With these techniques, different hardened layer thicknesses have been produced, for example, induction heating 
treatment can yield up to $3 \mathrm{~mm}$ thick hardened layers on AISI 1045 steel [1], plasma arc melting can produce $50 \mu \mathrm{m}$ hardened layers on 45 steel [2], and laser quenching (LQ) reported by Telasang et al. can yield $400 \mu \mathrm{m}$ hardened layers on the surface of H13 steel [3]. Although induction heating generates thick layers, only laser surface hardening generates uniform layers in complex geometries, thus gaining research attention due to its potential industrial use.

Laser surface hardening is a technique that has been used and investigated since the 1970s. However, research for industrial applications has mostly been conducted using $\mathrm{CO}_{2}$ lasers with power densities ranging from $10^{3}$ to $10^{5} \mathrm{~W} /$ $\mathrm{cm}^{2}$ [4]. In 2004, Kennedy reported that laser surface hardening could be a technology that can be applied at the industrial scale because of the increase in power density and the decrease in laser cost associated with the development of high-power diode lasers [5].

Although laser surface hardening is a technique under constant development, issues like variation in the surface morphology [6] and nonhomogeneity of the surface microstructure still persist [7]. The main reason for these issues is a large number of parameters involved in this technique such as the peak temperature, heating and cooling rates, and the lack of information regarding the evolution of the microstructure with time. Farshidianfar recently proposed a methodology for controlling the microstructure and geometry during the surface LQ process through real-time thermal monitoring using an infrared-based thermoimaging system [8]. This technique reduces the uncertainty of homogeneity in the properties achieved. Other studies tried to increase the reliability of laser surface hardening by investigating the effects of laser power and laser scanning speeds on the microstructure, hardening depth, and surface hardness of AISI low-carbon steel $[8,9]$ and by validating the use of diode lasers instead of $\mathrm{CO}_{2}$ lasers to harden AISI 1040 medium carbon steel $[9,10]$.

Most researchers suggest that only laser parameters affect the formation of an appropriate hardened layer; however, Yong et al. have demonstrated that, in a real manufacturing process, the manufacturing technique also plays an important role by studying the evolution of the microstructure and properties of nonquenched and tempered $1538 \mathrm{MV}$ steel. Using a crankshaft produced by the forging process, they demonstrated that the forging temperature and cooling process directly affect the precipitate size and dispersion after forging [11]. If the hardness of this steel is modified, then its fatigue limit would also change as reported by Li et al. [12].

Although many studies of laser surface hardening have been conducted on several types of steel, to the best of our knowledge, none of them aimed at comparing the tribological properties of laser-treated samples against those of samples treated by the conventional technique in order to understand to what extent the laser-treated parts could be reliable. The main motivation to avoid conventional quenching is the large amount of energy required to harden the entire part.

This work compares the tribological behaviors of $1538 \mathrm{MV}$ steel, chosen as a representative material used in the automotive industry, subjected to LQ and CQT. The variables studied include the friction coefficient, wear resistance, grain morphology, and hardness.

\section{Materials and Methods}

2.1. Sample Preparation. In this study, disks of $1538 \mathrm{MV}$ steel with $66 \mathrm{~mm}$ diameter, $5 \mathrm{~mm}$ thickness, and typical hardness of $23.31 \pm 1.75 \mathrm{HRC}$ were used as test samples. The composition of this material is shown in Table 1.

Before and after thermal treatment, all samples were ground with $\mathrm{SiC}$ sandpaper (grit numbers from 120 up to 600 ) in order to obtain surfaces with a roughness of $0.1 \pm 0.03 \mu \mathrm{m}$ measured using a roughness tester Mitutoyo SJ400.

\subsection{Conventional Thermal Treatment (Quenching-} Tempering). Three samples were quenched at the austenitization temperature $(1198 \mathrm{~K})$ for $4.5 \mathrm{~h}$ following the recommendation made in the ASM handbook [1]. The samples were fully immersed in a container in a reducing atmosphere, and the quenching was carried out in a commercially available quenching solution based on polyoxythylene glycol. Once treated, the samples were tempered at $523 \mathrm{~K}$ for $4 \mathrm{~h}$.

2.3. Laser Quenching. Laser thermal treatment was carried out using a $10 \mathrm{~kW}$ continuous-wave $1080 \mathrm{~nm}$ laser diode (Laserline model LDF 10.00-100). This device has a three Cartesian axes positioning system $(x, y$, and $z$ ) and an angular positioning system. The samples were placed perpendicular to the laser ( $z$ coordinate) over a pneumatic centering table. Programmed thermal treatment was carried out in a single step using a spot diameter of $3.5 \mathrm{~mm}$, sweeping a $30 \mathrm{~mm}$ band at a $168 \mathrm{~mm} / \mathrm{s}$ linear speed to achieve a recommended coverage rate of $5.04 \mathrm{~cm}^{2} / \mathrm{s}$ [13].

2.4. Metallographic Procedures. Small sections were cut out from the three disks to study the cross sections using optical microscopy. Grinding and polishing were carried out following the ASTM-E3-01 procedures. To expose the microstructure, the samples were etched using a $4 \%$ nital solution for $8 \mathrm{~s}$.

2.5. Measurement of Hardness. Hardness measurements were carried out using a hardness tester (8SSA-10287) with a $105 \mathrm{~kg}$ load and $10 \mathrm{~kg}$ preload on the following three types of samples: sample not subjected to thermal treatment, sample quenched with laser, and sample subjected to conventional quenching-tempering. Additionally, three microhardness profiles were obtained from the cross section of the sample subjected to laser treatment using a microhardness tester (Shimadzu, type M, no. 89188).

2.6. Estimation of the Friction Coefficient. Wear tests were carried out in dry conditions according to the ASTM G99 standard using a conventional tribometer (CSM, S/N 18-259) 
TABLE 1: Chemical composition of $1538 \mathrm{MV}$ steel (\%wt).

\begin{tabular}{cccccccccccccc}
\hline $\mathrm{Mn}$ & $\mathrm{C}$ & $\mathrm{Si}$ & $\mathrm{S}$ & $\mathrm{P}$ & $\mathrm{Ti}$ & $\mathrm{Cr}$ & $\mathrm{Cu}$ & $\mathrm{V}$ & $\mathrm{Ni}$ & $\mathrm{Sn}$ & $\mathrm{Mo}$ & $\mathrm{Al}$ & $\mathrm{Fe}$ \\
\hline 1.31 & 0.36 & 0.83 & 0.05 & 0.038 & 0.72 & 0.156 & 0.128 & 0.082 & 0.051 & 0.022 & 0.015 & 0.02 & Balance \\
\hline
\end{tabular}

and a $5 \mathrm{~mm}$ WC ball (1500 HV) arranged as shown in Figure 1. The chemical composition of this ball is Co matrix (5-7\%), other carbides ( 2\%), and WC (balance).

The following testing parameters were used: load of $5 \mathrm{~N}$, speed of $0.10 \mathrm{~m} / \mathrm{s}$, sliding distances of $100,200,300$, and $500 \mathrm{~m}$, track radii of $4,6,8$, and $10 \mathrm{~mm}$ for every sliding distance, relative humidity of $40 \pm 5 \%$, and room temperature of $292 \pm 2 \mathrm{~K}$.

2.7. Wear Mechanisms in the Sample and WC Steel Ball. Wear scars in the WC ball were evaluated through optical microscopy (OLYMPUS, PMG-3), and the wear mechanisms in all disks were evaluated using scanning electron microscopy (JEOL, JSM-6360LV).

2.8. Estimate of Wear Rate. Wear rate was estimated from the diameter of the wear scars generated on the surface of the WC ball and the track area in the steel disks. Lost volume for every sample and their counterpart can be calculated using the following equation $[14,15]$ :

$$
V=\frac{\pi b^{4}}{64 R}
$$

where $V$ is the lost volume, $b$ is the wear scar radius, and $R$ is the sphere radius for $b<<R$. by

Wear rate estimation is based on the Archard law given

$$
V=k s N
$$

where $V$ is the lost volume, $k$ is the wear constant, $s$ is the sliding distance, and $N$ is the applied load.

Hence, the wear rate was calculated as follows:

$$
k=\frac{\pi b^{4}}{64 R s N} .
$$

\section{Results and Discussion}

The micrographs obtained by optical microscopy of the untreated (RAW) sample (Figure 2(a)) reveal disperse perlitic and ferritic phases, while the micrograph of the laserquenched sample (Figure 2(b)) shows a layer of martensitic phase up to a depth of $760 \mu \mathrm{m}$ from the surface followed by a structure similar to that of the original substrate. The sample subjected to CQT shows a continuous martensitic phase (Figure 2(c)). Further, the micrographs, with a higher magnification, in Figures 3(a) and 3(d) clearly show the typical microstructure of $1538 \mathrm{MV}$ steel consisting of perlitic and ferritic phases; meanwhile, for the laser-quenched zone of the LQ sample, it is possible to see a martensitic phase with a regular grain size (Figures $3(\mathrm{~b})$ and $3(\mathrm{e})$ ), which could be attributed to the uniform cooling of the material during

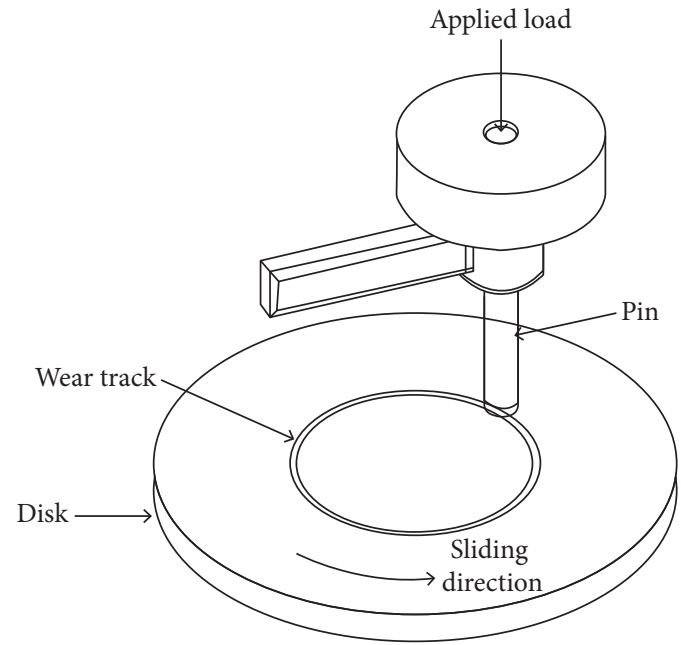

FIGURE 1: Schematic representation of a ball-on-disk test of the tribo-pair of AISI $1538 \mathrm{MV}$ steel disk and WC ball.

the laser quenching process. On the other hand, Figures 3(c) and 3(f) show grains of martensitic phase surrounded by small quantities of the retained austenite phase for the CQT sample [16]. Also, XRD patterns corroborate the presence of the ferritic and martensitic phases (see Figure 4).

The initial hardness of the sample was $24.12 \pm 1.2 \mathrm{HRC}$, and the surface hardness increased to $51.5 \pm 2.5$ and $52.7 \pm 1$ $\mathrm{HRC}$, respectively, after laser quenching treatment and conventional treatment.

The average microhardness measured ( 3 measurements at the same depth) over the cross section of the LQ samples was characterized by decreasing hardness from the surface towards the center of the substrate. As expected, a sudden drop in hardness at the end of the martensitic zone (760 $\mu \mathrm{m}$ ) was observed for the LQ sample (see Figure 5(a)); this type of behavior is due to the type of atmosphere (air) used for the treatment, as demonstrated recently by Maharjan et al. [17]. On the other hand, as shown in Figure 5(b), the hardness of the CQT sample remained similar beyond a depth of $1 \mathrm{~mm}$.

The lowest friction coefficient during the tests was obtained for LQ samples, while the highest value was obtained for the CQT samples. The RAW sample showed a constant (0.37) average friction coefficient up to a sliding distance of $200 \mathrm{~m}$, and then, this value significantly increased (up to 0.45 ) for a distance of $500 \mathrm{~m}$. The average friction coefficient for the CQT samples remained almost constant (0.43-0.45) for all sliding distances with only a small increment being observed. Finally, the LQ samples showed the lowest friction coefficient with similar friction coefficient values $(0.295)$ for the sliding distances of 100 and $200 \mathrm{~m}$, and then, the friction coefficient increased slightly and remained similar (0.361) for the 300 and $500 \mathrm{~m}$ distances, as shown in Figure 6. 


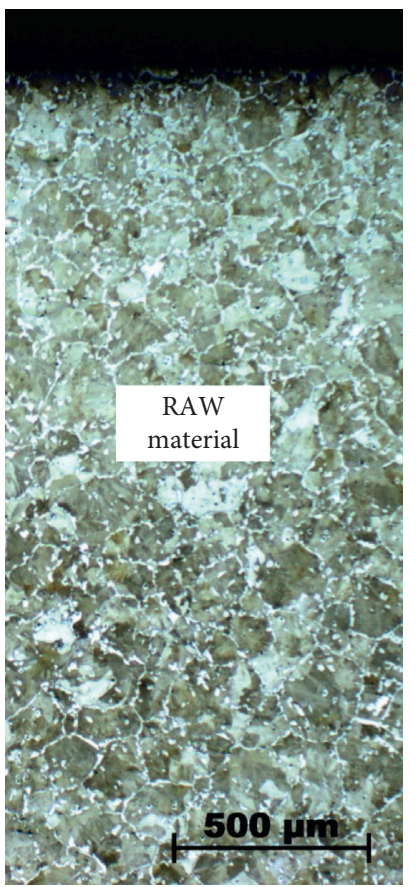

(a)

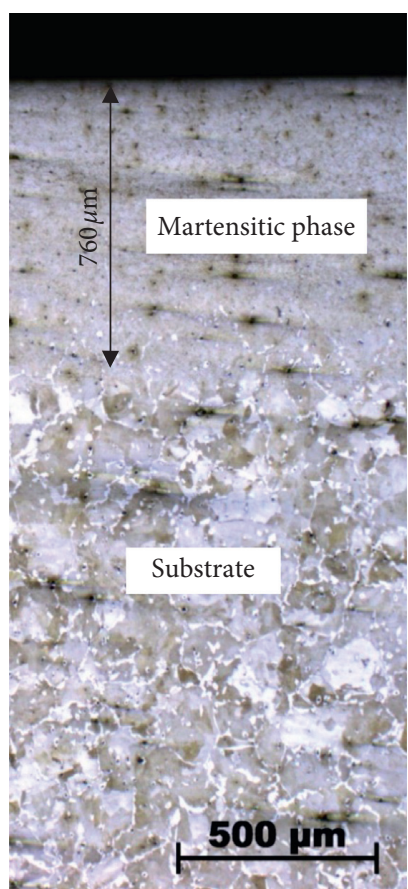

(b)

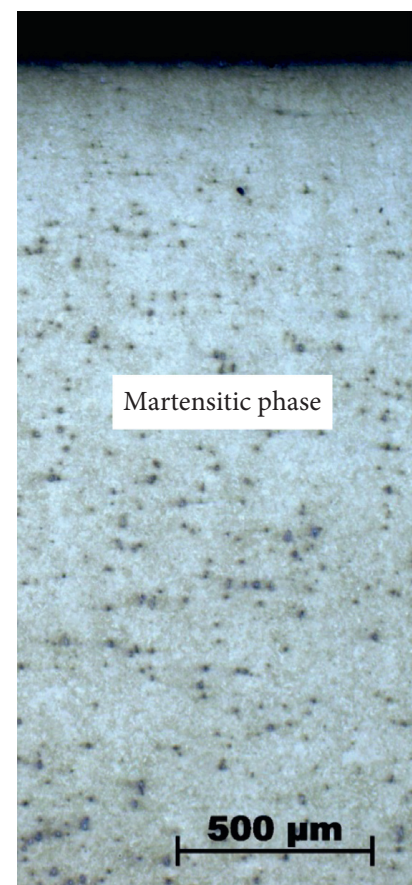

(c)

FIGURE 2: Comparison of the cross section of samples: (a) RAW sample, (b) LQ sample, and (c) CQT sample.

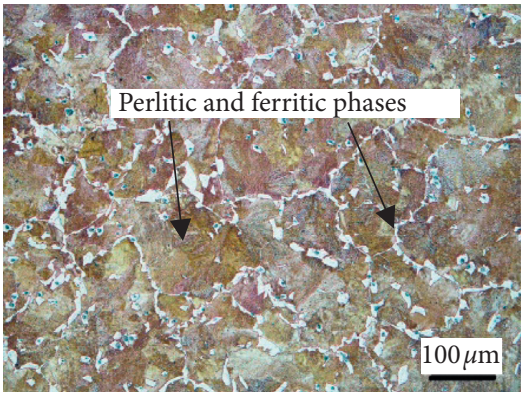

(a)

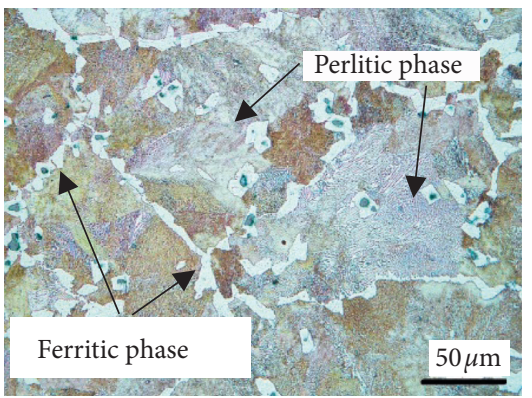

(d)

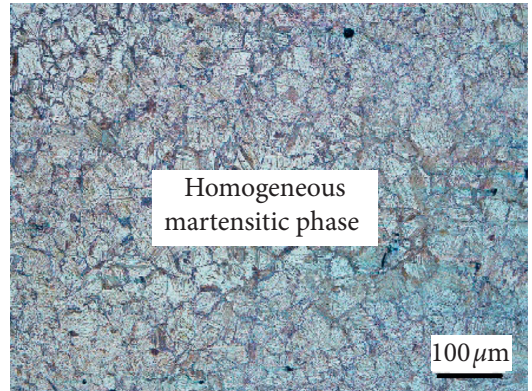

(b)

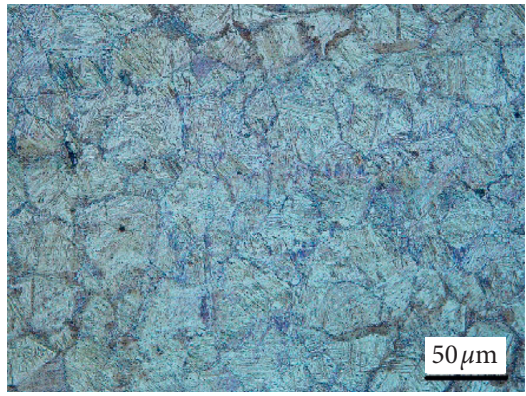

(e)

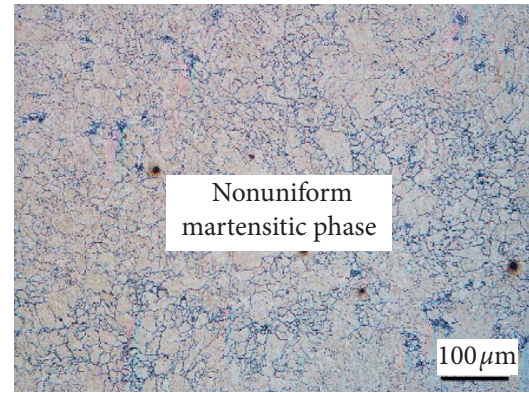

(c)

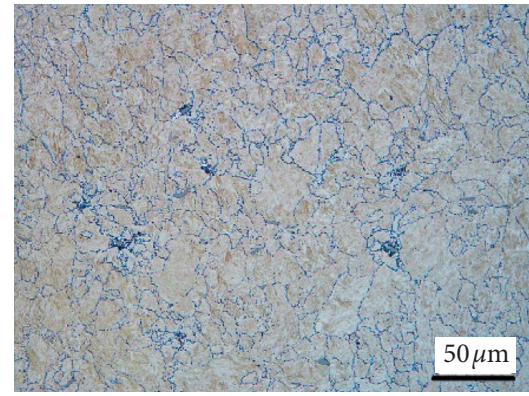

(f)

FIGURE 3: Grain morphology of the longitudinal section for (a) RAW sample, (b) LQ sample, and (c) CQT sample. Higher magnification images show (d) perlitic and ferritic phases for RAW material, (e) homogeneous martensitic phase, and (f) nonuniform martensitic phase.

The friction coefficient behavior of all samples is characterized by a running in zone and a steady-state zone. The running in zone appears approximately during the first $50 \mathrm{~m}$; here, the friction coefficient reaches a maximum value and then decreases to a steady value. This behavior could be related to hardness and grain morphology. Therefore, in the running in zone, RAW samples have the greatest friction coefficient, possibly related to severe plastic deformation, 


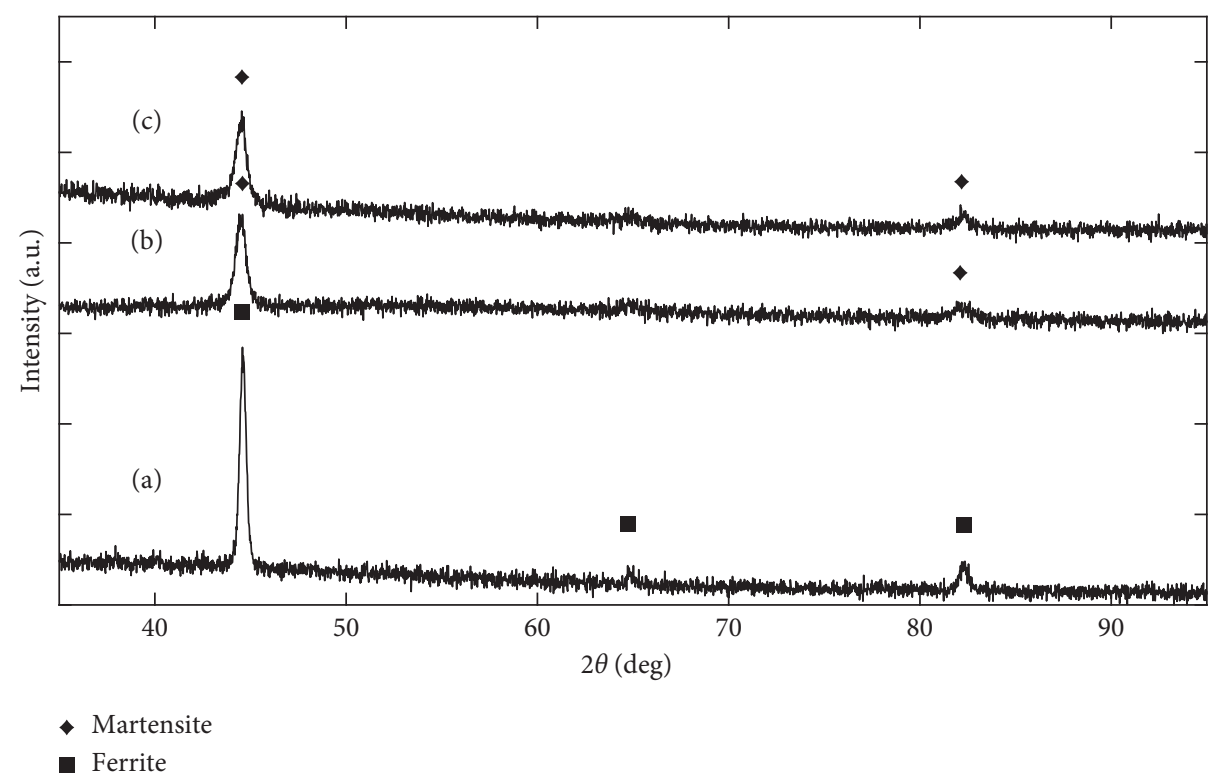

FIGURE 4: XRD patterns of samples surface for different conditions: (a) RAW, (b) CQT, and (c) LQ.

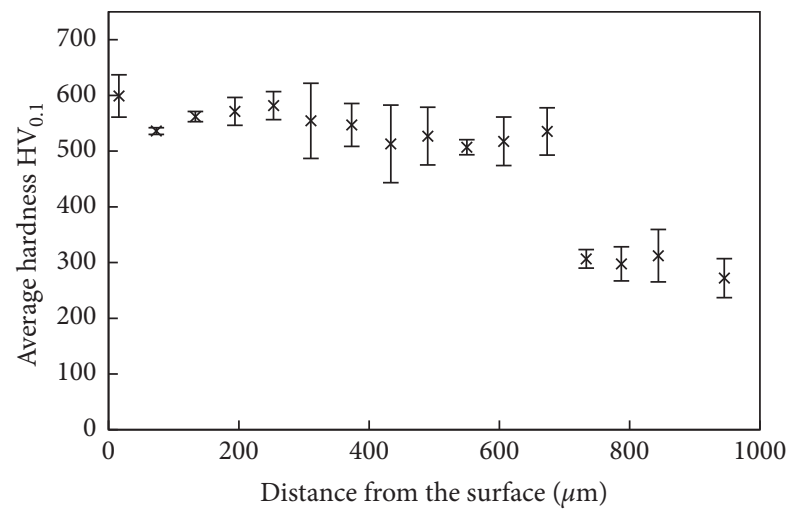

(a)

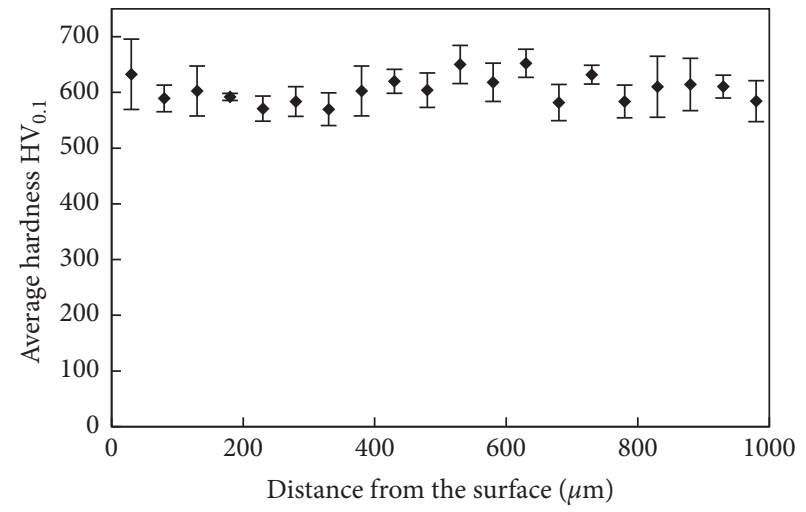

(b)

FIGURE 5: Microhardness profiles of the cross section of (a) LQ and (b) CQT samples.

while CQT has a moderate friction coefficient value due to its higher hardness and amorphous grain size distribution and LQ has the lowest friction coefficient value due to its uniform grain size and high hardness. Then, a steady value appears for RAW samples at $100 \mathrm{~m}$, a slightly growing behavior for LQ samples from $150 \mathrm{~m}$, and an irregular behavior for CQT samples (see Figure 7). Despite the small thickness of the treated layer, LQ samples consistently show the smallest values, which are 20 to $30 \%$ smaller than those of CQT samples.

For the maximum distance $(500 \mathrm{~m})$, the friction coefficient was found to be $0.458 \pm 0.058,0.456 \pm 0.063$, and $0.361 \pm 0.056$ for RAW, CQT, and LQ samples, respectively.

As part of the wear studies, the surface of WC balls was observed by optical microscopy. These surfaces are characterized by grooves (ploughing effects) caused by the presence of debris, which are particles detached from the sample surface due to sliding wear $[18,19]$. The sliding direction plays an important role in the adhesion of particles in the case of RAW and CQT samples (Figures 8(a) and $8(\mathrm{~b})$ ), for which the material concentrates at the back face of the ball, while it did not occur for LQ samples (Figure 8(c)). The wear scar diameter was 540,430 , and $385 \mu \mathrm{m}$ for RAW, CQT, and LQ samples, respectively. Since both wear rate and friction coefficient are lower for LQ samples compared to those of CQT samples, a longer lifespan for parts treated with laser is expected, as previous reports suggest [20-22].

Scanning electron micrographs (SEMs) demonstrate the presence of adhesion particles in all samples (Figure 9), and macro- and microplastic deformation for RAW and treated samples, respectively (Figures 9(a)-9(c)). Additionally, the presence of $\mathrm{C}, \mathrm{Fe}, \mathrm{Mn}, \mathrm{O}$, and $\mathrm{W}$ elements, in both the sample and sphere, was determined by energy-dispersive $\mathrm{X}$-ray spectroscopy, suggesting that the plastic deformation and adhesion are caused by the exchange of debris between both parts of the tribo-pair. These particles could cause 


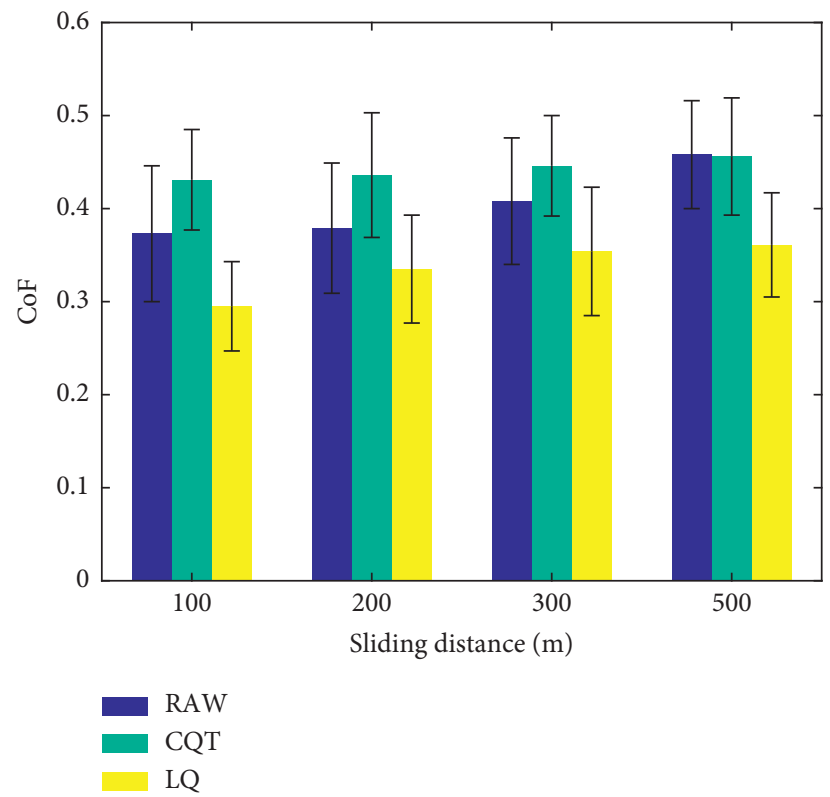

FIGURE 6: Friction coefficient of samples under different conditions and sliding distances.

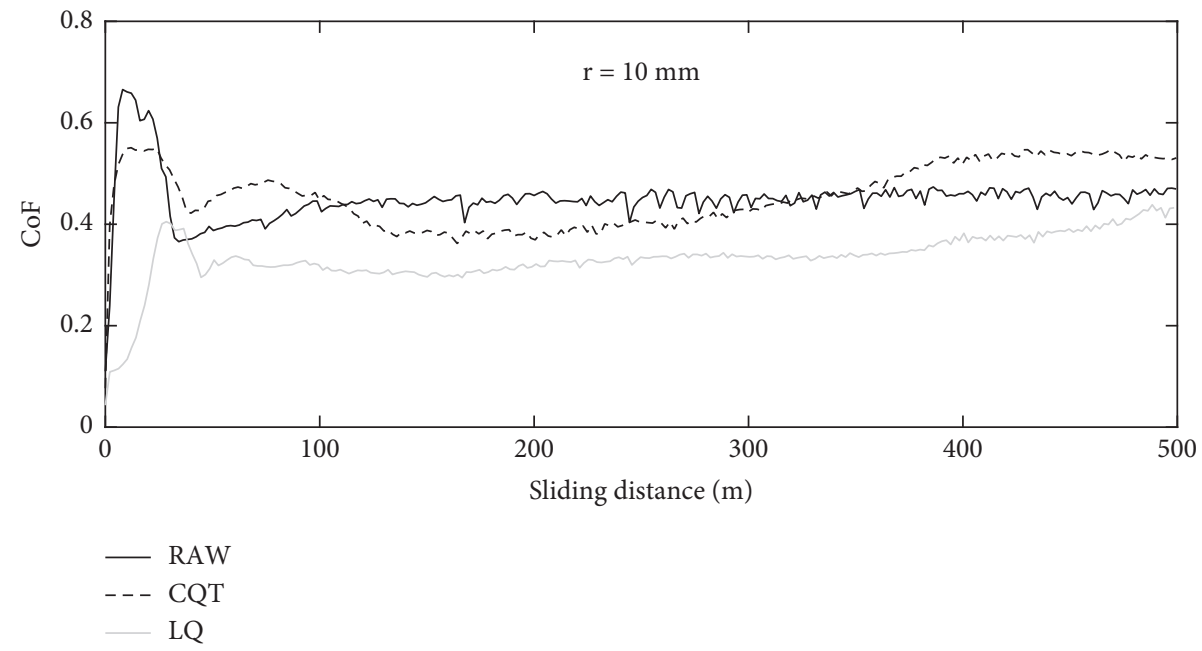

Figure 7: Friction coefficient for $500 \mathrm{~m}$ sliding distances. Pin-on-disk tests for RAW, CQT, and LQ samples.

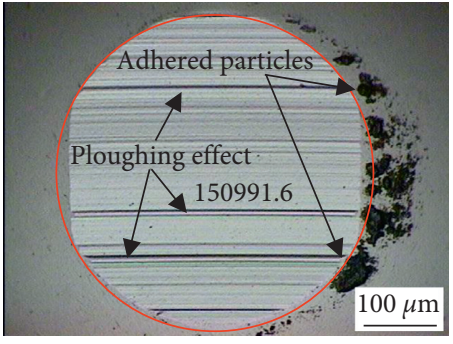

(a)

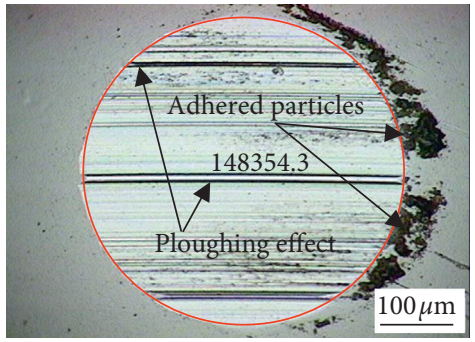

(b)

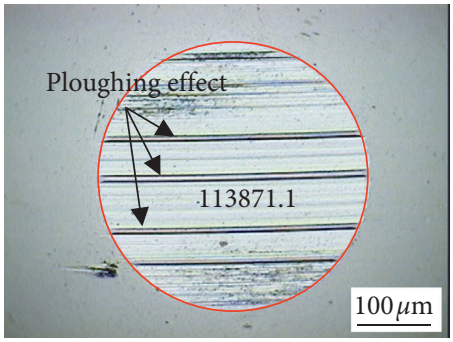

(c)

Figure 8: Wear scars on the WC ball used to test samples: (a) RAW, (b) CQT, and (c) LQ for a sliding distance of $500 \mathrm{~m}$. Notice the lack of adhered particles for LQ condition. 

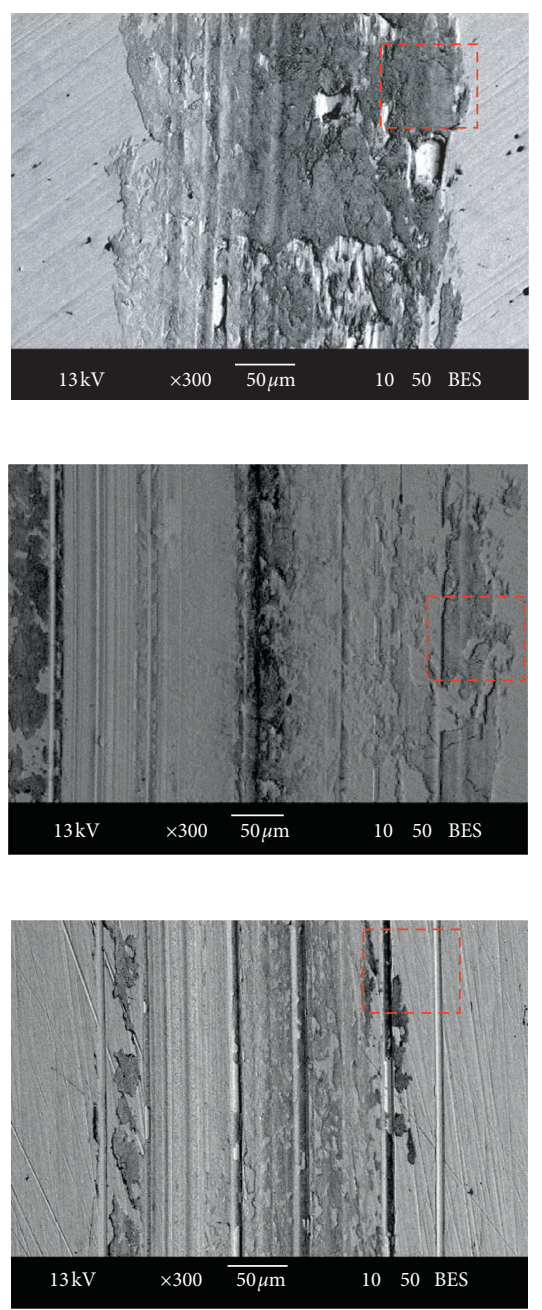
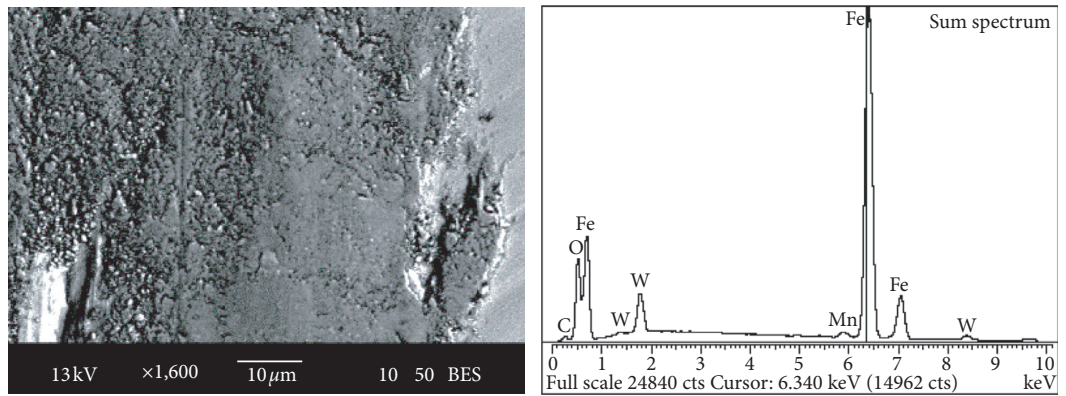

(a)
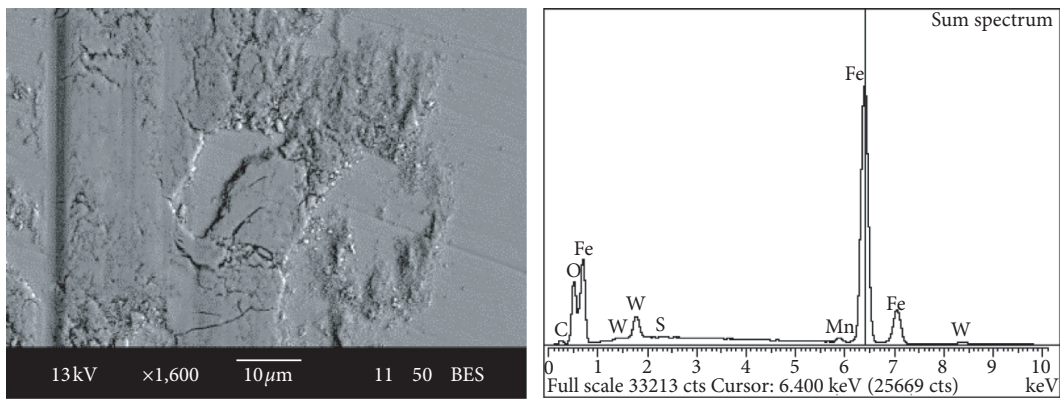

(b)
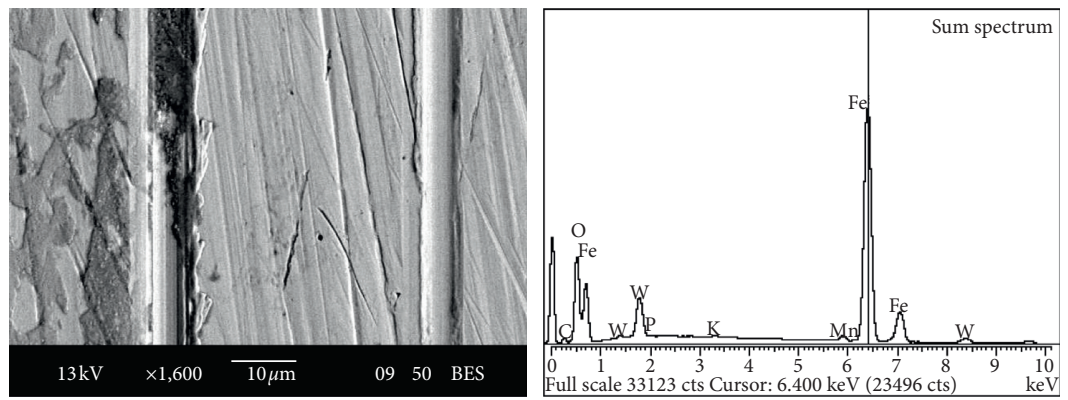

(c)

FIGURE 9: SEM images (first column) of the wear tracks generated on the surface of the steel disk at a distance of $500 \mathrm{~m}$ enlarged image (second column) of the region enclosed in red square, and the corresponding EDS profiles (third column). First, second, and third rows correspond to (a) RAW, (b) CQT, and (c) LQ samples.

severe wear in lubricated conditions when the contact is subjected to high loads, due to the burst of the film [23]. Since $1538 \mathrm{MV}$ steel is used for components subjected to high loads and temperatures (such as crankshafts), special care should be taken when using the LQ technique to treat downsized mechanical components.

Figure 10 shows the average wear rates of samples for different sliding distances. RAW samples always have the highest average wear rate with a maximum value of $2.4 \times 10^{-5} \mathrm{~mm}^{3} / \mathrm{N} \cdot \mathrm{m}$ for a sliding distance of $500 \mathrm{~m}$, while the opposite is true for LQ samples with a value of $0.4 \times 10^{-5} \mathrm{~mm}^{3} / \mathrm{N} \cdot \mathrm{m}$ for the same distance. The wear rates of treated samples vary from 4 to $24 \%$ for distances up to $300 \mathrm{~m}$, and then the values diverge and show a marked difference ( $43 \%$ for $500 \mathrm{~m}$ ). In general, RAW samples have a threefold higher rate than the treated samples, from which it can be inferred that samples subjected to laser treatment outperform those subjected to conventional treatment. This phenomenon could be attributed to severe plastic deformation, which causes a greater material removal from RAW samples and a similar amount of material from LQ and CQT samples.

Profilometry results show different wear mechanisms for the samples. For the RAW sample, plastic deformation is predominant due to the high ductility of the RAW material, and the scratch depth reaches up to $4 \mu \mathrm{m}$ and the wear track is slightly larger $(593 \mu \mathrm{m})$ compared to the ball scar diameter $(540 \mu \mathrm{m})$, as shown in Figure 11(a). In CQT and LQ samples, plastic flow towards the edges does not occur as a consequence of the surface hardness. Therefore, the loss of material is negligible, the wear track depth remained under $1 \mu \mathrm{m}$, and the wear track was same and wear scar diameter was similar (see Figures 11(b) and 11(c)).

It is desirable that surface-hardened products withstand high contact pressures for a prolonged lifespan with minimum wear and friction. If the CQT technique is to be replaced by the LQ method, properties related to wear resistance should be maintained within an acceptable level. In 


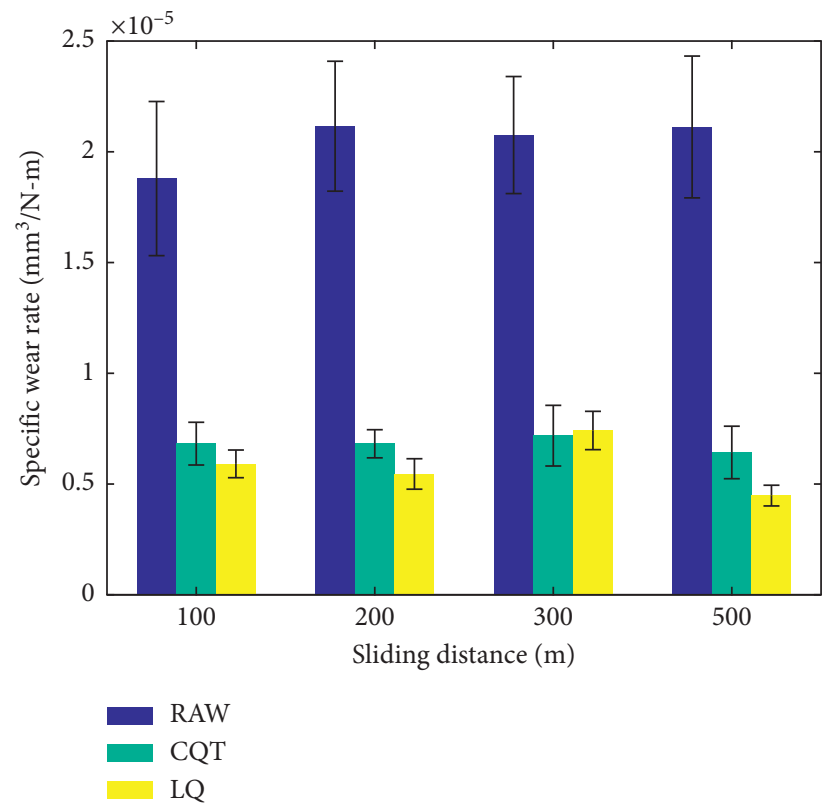

Figure 10: Specific wear rate and sliding distance for different testing conditions.

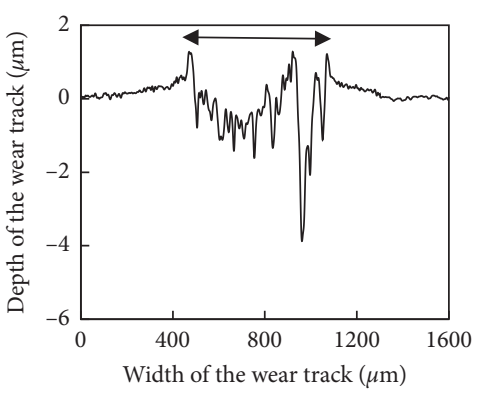

(a)

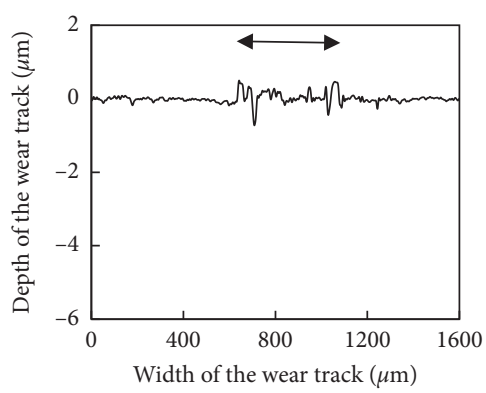

(b)

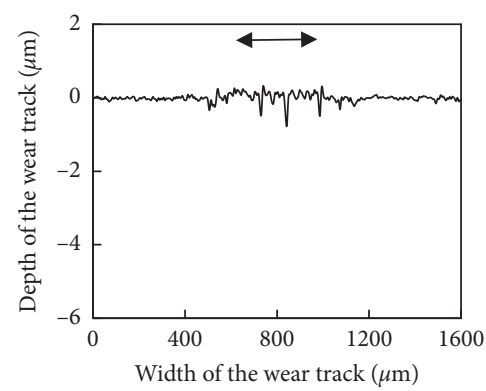

(c)

FIGURe 11: Profilometry plots of wear tracks for (a) RAW, (b) CQT, and (c) LQ samples.

this work, we observed that the hardness of LQ-treated $1538 \mathrm{MV}$ steel can increase by $113 \%$ up to a depth of $760 \mu \mathrm{m}$, which is only a hardness value similar compared with CQT. This implies that the contacting pressure that both LQ- and CQT-treated samples can withstand is expected to be similar.

\section{Conclusions}

A comparison of laser quenching versus conventional quenching and tempering is presented in this study. Results obtained show that LQ-treated samples have similar tribological properties to the CQT samples, hence making the former a good option to be adopted as an ecologically friendly process.

LQ samples consistently showed reduced wear rate and average friction coefficient; in practical terms, these features are advantageous because they are directly related to the lifespan increase of machine components as literature shows.
Even when hardness is similar for treated samples using a conventional and a laser technique, the wear mechanisms are more severe for the former process, thus causing a deeper wear track that originates greater damage by promoting microfatigue initiation phenomenon.

\section{Data Availability}

The data used to support the findings of this study are available from the corresponding author upon request.

\section{Conflicts of Interest}

The authors declare that there are no conflicts of interest regarding the publication of this paper.

\section{Acknowledgments}

The authors would like to thank CINVESTAV Queretaro for their kind support in obtaining some metallographic images 
and the XRD patterns. The authors gratefully acknowledge the financial support from the Mexican National Council for Science and Technology (CONACYT).

\section{References}

[1] ASM International, ASM Handbook Volume 4: Heat Treating; American Society for Metals, ASM International: Metals Park, OH, USA, 1991.

[2] M. Yan and W. Z. Zhu, "Surface treatment of 45 steel by plasma-arc melting," Surface and Coatings Technology, vol. 91, no. 3, pp. 183-191, 1997.

[3] G. Telasang, J. Dutta Majumdar, G. Padmanabham, M. Tak, and I. Manna, "Effect of laser parameters on microstructure and hardness of laser clad and tempered AISI H13 tool steel," Surface and Coatings Technology, vol. 258, pp. 1108-1118, 2014.

[4] J. Grum, "Laser surface hardening," in Encyclopedia of Tribology, Q. J. Wang and Y.-W. Chung, Eds., Springer US, Berlin, Germany, pp. 1948-1962, 2013.

[5] E. Kennedy, G. Byrne, and D. N. Collins, "A review of the use of high power diode lasers in surface hardening," Journal of Materials Processing Technology, vol. 155-156, pp. 1855-1860, 2004.

[6] M. Hua, S. Dao, T. M. Shao, and H. Y. Tam, "Surface transformation of DF-2 steel after continuous mode laser irradiation," Journal of Materials Processing Technology, vol. 192-193, pp. 89-96, 2007.

[7] P. D. Babu, K. R. Balasubramanian, and G. Buvanashekaran, "Laser surface hardening: a review," International Journal of Surface Science and Engineering, vol. 5, no. 2-3, pp. 131-151, 2011.

[8] M. H. Farshidianfar, A. Khajepouhor, and A. Gerlich, "Realtime monitoring and prediction of martensite formation and hardening depth during laser heat treatment," Surface and Coatings Technology, vol. 315, pp. 326-334, 2017.

[9] T.-P. Hung, H.-E. Shi, and J.-H. Kuang, "Temperature modeling of AISI 1045 steel during surface hardening processes," Materials, vol. 11, no. 10, p. 1815, 2018.

[10] S. Guarino, M. Barletta, and A. Afilal, "High Power Diode Laser (HPDL) surface hardening of low carbon steel: fatigue life improvement analysis," Journal of Manufacturing Processes, vol. 28, pp. 266-271, 2017.

[11] Y. Yong, L. Zhou, D. Fu, P. Jiang, and X. Ren, "Microstructure and property evolution of the $1538 \mathrm{MV}$ non-quenched and tempered steel for a crankshaft during the forging process," Materiali in Tehnologije, vol. 52, no. 5, pp. 547-553, 2018.

[12] J. Li, Y. Qiu, H. Wang, and Z. Wang, "Evaluation of the methods for estimating the fully reversed unnotched fatigue limits of steels," Advances in Materials Science and Engineering, vol. 2019, 11 pages, 2019.

[13] J. F. Ready, "Chapter 15-applications for surface treatment," Industrial Applications of Lasers, Academic Press, Cambridge, MA, USA, pp. 373-383, 1997.

[14] I. Hutchings, Tribology: Friction and Wear of Engineering Materials, Butterworth-Heinemann, Oxford, UK., 1st edition, 1992.

[15] A. W. B. G. W. Stachowiak, Boundary and Extreme Pressure Lubrication, Engineering Tribology, Butterworth-Heinemann, Oxford, UK., 3rd edition, 2006.

[16] X. L. Ping, H. G. Fu, K. M. Wang et al., "Effect of laser quenching on microstructure and properties of the surface of track materials," Surface Review and Letter, vol. 25, pp. 1-10, 2018 .
[17] N. Maharjan, W. Zhou, and N. Wu, "Direct laser hardening of AISI 1020 steel under controlled gas atmosphere," Surface and Coatings Technology, vol. 385, p. 125399, 2020.

[18] E. Hernández-Sánchez, J. C. Velázquez, J. L. Castrejón-Flores et al., "Tribological behavior of borided AISI 316L steel with reduced friction coefficient and enhanced wear resistance," Materials Transactions, vol. 60, no. 1, pp. 156-164, 2019.

[19] R. Carrera-Espinoza, U. Figueroa-López, J. Martínez-Trinidad, I. Campos-Silva, E. Hernández-Sánchez, and A. Motallebzadeh, "Tribological behavior of borided AISI 1018 steel under linear reciprocating sliding conditions," Wear, vol. 362-363, pp. 1-7, 2016.

[20] Z. Chen, Q. Zhu, J. Wang, X. Yun, B. He, and J. Luo, "Behaviors of $40 \mathrm{Cr}$ steel treated by laser quenching on impact abrasive wear," Optics \& Laser Technology, vol. 103, pp. 118-125, 2018.

[21] S. Da Sun, D. Fabijanic, C. Barr et al., "In-situ quench and tempering for microstructure control and enhanced mechanical properties of laser cladded AISI 420 stainless steel powder on $300 \mathrm{M}$ steel substrates," Surface and Coatings Technology, vol. 333, pp. 210-219, 2018.

[22] Y. Li, W. Li, W. Liu et al., "The austenite reversion and coprecipitation behavior of an ultra-low carbon medium manganese quenching-partitioning-tempering steel," Acta Materialia, vol. 146, pp. 126-141, 2018.

[23] A. Rosenkranz, P. G. Grützmacher, K. Murzyn, C. Mathieu, and F. Mücklich, "Multi-scale surface patterning to tune friction under mixed lubricated conditions," Applied Nanoscience, vol. 25, 2019. 